\title{
La Sociedad Latinoamericana de Nefrología e Hipertensión y los retos de la enfermedad renal crónica en nuestra región
}

\author{
Alfonso M. Cueto-Manzano ${ }^{1,2 *}$ \\ ${ }^{1}$ Sociedad Latinoamericana de Nefrología e Hipertensión 2017-2019; ²Unidad de Investigación Biomédica 02, Hospital de Especialidades, Centro \\ Médico Nacional de Occidente, Instituto Mexicano del Seguro Social, Guadalajara. Jalisco, México
}

\section{Resumen}

La enfermedad renal crónica (ERC) en Latinoamérica es un grave problema de salud pública que requiere esfuerzos de todos los sectores de la población. Los principales retos en la atención de la ERC hoy día son:

- Lograr cobertura universal de las terapias de reemplazo renal (TRR) para que todos los pacientes que las requieren las reciban efectivamente.

- Lograr una prevalencia mínima de TRR de 700 pacientes por millón de población en todos los países. Para ello, es necesario incentivar el uso de TRR costo-efectivas: trasplante renal y diálisis peritoneal.

- Estimular la mayor formación de nefrólogos para alcanzar una tasa de 20 por millón de población, pues la participación de este especialista es indispensable.

- Estimular la investigación local, regional y multicéntrica sobre diversas áreas de la ERC, incluyendo sus causas más comunes y las particulares que afectan a ciertas regiones. Sus resultados contribuirán a resolver nuestros propios problemas.

- Contrarrestar efectivamente la ERC, por lo que los esfuerzos deben enfocarse en la prevención, diagnóstico y tratamiento de ERC temprana. Las intervenciones educativas para el equipo de salud y los pacientes son de las mejores herramientas para este fin. Involucrar al personal multidisciplinario en modelos de intervención múltiple debe privilegiarse cuando sea posible.

- Conocer la magnitud real de la ERC, tomar decisiones para contrarrestarla y evaluar resultados de posibles acciones, por lo que es indispensable crear registros nacionales de ERC en todos los países que aún carezcan de ellos, e incrementar la calidad de los ya existentes.

Palabras clave: SLANH. Enfermedad renal crónica. Latinoamérica.

\section{The Latin American Society of Nephrology and Hypertension and the challenges of chronic kidney disease in our region}

\section{Abstract}

Chronic kidney disease (CKD) in Latin America is a huge public health problem requiring efforts from all the population sectors. Currently, the main challenges of the CKD attention in our region are:

- Establish universal coverage of renal replacement therapies (RRT) for all patients requiring treatment.

- $\quad$ Achieve a minimal RRT prevalence of 700 patients per million population in all countries. To get this goal, it is necessary to stimulate the use of cost-effective RRT: kidney transplant and peritoneal dialysis.

\section{Correspondencia:}

*Alfonso M. Cueto-Manzano

E-mail: a_cueto_manzano@ hotmail.com

(11741. d Access bajo la licencia CC BY-NC-ND (http://creativecommons.org/licenses/by-nc-nd/4.0/)
Fecha de recepción: 19-11-2018

Fecha de aceptación: 26-12-2018

DOI: $10.24875 /$ NEFRO. 18000053
Disponible en internet: 09-08-2019

Nefro Latinoam. 2019;16:13-19 www.nefrologialatinoamericana.com 
Nefro Latinoam. 2019;16

- Stimulate the formation of higher numbers of nephrologists to reach the rate of 20 per million population, as participation of this specialist is crucial.

- Encourage local, regional and multicentric research on several areas of CKD, including the most common and particular etiology affecting some specific regions. Its results will contribute to solve our own problems.

- In order to effectively counteract CKD, efforts should be focused on prevention, diagnosis and treatment of early CKD. Educative interventions addressed to the health team and patients are the best tools. Involvement of multidisciplinary health personnel in multiple intervention models must be privileged whenever possible.

- Create national CKD registries in all the countries still missing them, and increase the quality of those already existing are key to know the real magnitude of the problem, take appropriate decisions against it, and evaluate results of any action put in place.

Key words: SLANH. Chronic kidney disease. Latin America.

\section{Introducción}

Latinoamérica es una extensa región del mundo de poco más de 19 millones de $\mathrm{km}^{2}$ y con una población de 638 millones, que crece a un ritmo promedio del $1 \%$ anual ${ }^{1}$. Para muchos, los 20 países principales que la componen tienen como única característica en común el haber sido colonias y haber heredado la lengua de España y Portugal. Sin embargo, a pesar de las enormes diferencias entre países (e incluso dentro de un mismo país), la región comparte algunas características que ayudan a explicar por qué la enfermedad renal ocupa el lugar actual en América Latina, y que a su vez reflejan indudables progresos en muchas áreas pero también innegables rezagos en otras. Aunque en promedio ha habido mayor cobertura de servicios públicos a la población (p. ej., agua potable al 95\% de la población y drenaje público al $83 \%)^{2}$ y se ha logrado disminuir el analfabetismo (aunque a niveles aún no óptimos del $9 \%)^{3}$, el índice de desarrollo humano todavía no es alto $(0.75)^{4}$. En términos de macroeconomía, el producto interno bruto (PIB) de la región no es despreciable (USD 5,954 billones) y, aunque lento, crece a un ritmo de $1.7 \%$ al año, mientras que el PIB per capita hoy día es de USD $8,324^{5}$. Sin embargo, el grave problema de Latinoamérica es la injusta distribución de la riqueza, pues mientras que en esta zona viven algunas de las personas o familias más ricas del mundo, el $26 \%$ de los pobladores viven en la pobreza (pero en algunos países excede al $40 \%)^{5}$. Además de estas situaciones desventajosas en materia de economía, varias condiciones en la esfera de los servicios de salud no han logrado ser superadas. La expectativa promedio de vida al nacer es ahora de 75.8 años y, consecuentemente, la población de más de 60 años ya es del $9 \%{ }^{1}$. Aunque la mortalidad infantil se ha reducido a 13/1,000 nacidos vivos y el bajo peso al nacer $(<2,500 \mathrm{~g})$ al $8 \%$, aún no son cifras ideales. Si bien se ha reducido la desnutrición en la población general a un $6.2 \%{ }^{5}$, ahora uno de los problemas de salud más graves son el sobrepeso y la obesidad, que juntos llegan al $58 \%$ en nuestra región ${ }^{1}$. El cuadro anterior se agrava cuando se considera que el porcentaje del PIB dedicado a la salud es particularmente bajo (3.7\%) y el personal de salud (médicos y enfermeras) también es subóptimo ${ }^{1}$. Más aún, el número de nefrólogos, aunque muy heterogéneo en la región, en promedio es bajo (16 nefrólogos pmp) ${ }^{6}$, sobre todo en Centroamérica, México, Colombia, Chile, Bolivia, Paraguay y Ecuador. Esta cifra está por debajo de la meta mínima propuesta por la Sociedad Latinoamericana de Nefrología e Hipertensión (SLANH) para Latinoamérica, que es de 20 nefrólogos pmp; solo Puerto Rico, Cuba, Argentina, Brasil y Uruguay rebasan esta cifra ${ }^{6}$.

\section{Las enfermedades crónicas no transmisibles y la enfermedad renal crónica como causas de muerte}

La insuficiencia renal tiene una enorme importancia para nuestra zona porque, en primer lugar, Latinoamérica tiene la tasa de mortalidad por enfermedad renal crónica (ERC) más alta de todo el mundo, y dentro de nuestra región, la ERC es la segunda causa más importante de años de vida perdidos ${ }^{7}$. Según los datos del Institute for Health Metrics and Evaluation de EE.UU., las enfermedades crónicas no transmisibles (ECNT) son, con mucho, la principal causa de mortalidad en todo el mundo, seguidas por las enfermedades transmisibles y la violencia y accidentes ${ }^{8}$. De las ECNT, las principales causas de mortalidad son las enfermedades cardiovasculares, pero la diabetes y la ERC también son causas muy relevantes. Cuando se analiza solo la región latinoamericana, las ECNT siguen siendo las principales causas de mortalidad, pero la ERC y la diabetes incrementan en más del doble su 
presencia ${ }^{8}$. Más aún, si se revisan las causas de años de vida perdidos por discapacidad, la participación de las ECNT también son las principales causas, y la ERC y la diabetes juegan un papel más o menos similar en magnitud que como causas de muerte; sin embargo, cuando se considera solo a América Latina, su frecuencia se incrementa aún más notablemente como causas de años perdidos por discapacidad ${ }^{8}$.

\section{Incidencia y prevalencia de la enfermedad renal crónica}

Algunos países latinoamericanos comparten sus datos con el United States Renal Data System (USRDS) ${ }^{9}$, y en la comparación internacional del reporte de 2018 de este registro muestran una gran variabilidad en sus tasas de incidencia de pacientes en terapia de reemplazo renal (TRR), que van desde las más altas como en el caso de México (debe aclararse que México no tiene un registro nacional de diálisis y, en su lugar, el Registro Estatal de Diálisis y Trasplante del Estado de Jalisco aporta sus datos), pasan por tasas intermedias como las de Guatemala, Brasil, Chile, Uruguay y Argentina, y llegan hasta cifras de las más bajas como las de Colombia y Perú. En un análisis más completo con los datos del Registro Latinoamericano de Diálisis y Trasplante $(R L A D T)^{6}$, en funcionamiento desde 1991, se puede observar que en el reporte más reciente de 2016, la incidencia promedio en Latinoamérica es de 162 pacientes por millón de población (ppmp), pero de nuevo muestra gran heterogeneidad. Los países con mayor incidencia son Puerto Rico (419 ppmp), Jalisco (411 ppmp), Honduras (260 ppmp) y El Salvador (227 ppmp). Los dos últimos recientemente han aparecido dentro de los países con más pacientes nuevos en TRR, mientras que los países con menor incidencia de TRR son Colombia (79 ppmp), Perú (71 ppmp), Paraguay (46 ppmp) y República Dominicana (15 ppmp) ${ }^{6}$. Por otro lado, mientras que algunos países han empezado a encontrar una meseta en su crecimiento en la tasa de incidencia, países como Chile y México muestran aún un constante (y preocupante) crecimiento de la misma ${ }^{9}$.

Ahora bien, comparando los países latinoamericanos con el resto del mundo, de acuerdo a los datos del US$\mathrm{RDS}^{9}$, también se observa una gran variabilidad en términos de prevalencia, con países con tasas de las más elevadas (México y Chile), intermedias (Uruguay, Argentina y Brasil) y bajas (Colombia, Perú y Guatemala). En el RLADT ${ }^{6}$, la prevalencia promedio en la zona fue de 778 ppmp en 2016, con Puerto Rico (2,129 ppmp),
México (1,558 ppmp), Chile (1,470 ppmp) y Uruguay (1,123 ppmp) como los países con mayores tasas, y Honduras (289 ppmp), Paraguay (271 ppmp), Costa Rica (139 ppmp) y El Salvador (104 ppmp) con las menores tasas. Asimismo, el crecimiento observado de la prevalencia también ha sido constante por más de 30 años, con un crecimiento anual aproximado del $10 \%$.

Lo que ocurre en relación con los trasplantes en nuestra zona también muestra un patrón de gran variabilidad, pues de nuevo se observan países con gran actividad de trasplante renal, como Uruguay, Brasil, Argentina, México, Puerto Rico y Costa Rica, y otros prácticamente no reportan actividad, como Perú, República Dominicana y Nicaragua. Aunque la tasa de trasplante ha ido incrementándose de manera constante a través de los años, aún no es suficiente para cubrir las necesidades y listas de espera en cada país ${ }^{6}$.

\section{Reto: Lograr la cobertura universal de las terapias de reemplazo renal y el uso de las terapias más costo-efectivas}

La gran heterogeneidad en términos de incidencia y prevalencia de las TRR arriba mostrada puede explicarse por la presencia diferenciada de varios factores de riesgo para ERC en cada país, si bien es conocido que muchos factores de riesgo son tan frecuentes en Centroamérica (que reporta las menores tasas de incidencia y prevalencia) como en algunos países de Sudamérica y México. Es más probable que esta variabilidad se deba a la injusta distribución de la riqueza que priva en esta zona, a la presencia de sistemas de salud fragmentados (muchas veces sin cobertura universal), al bajo porcentaje del PIB invertido en salud y a la manera particular en que cada país afronta la enfermedad ${ }^{7}$. Una cosa, sin embargo, parece clara en Latinoamérica: muchos pacientes que requieren TRR, en realidad, no la reciben.

Por tanto, el primer reto que Latinoamérica en su conjunto debe afrontar es lograr que todos los países tengan una cobertura universal. Un indicador con el que podría medirse que esto se va logrando es el incremento en la prevalencia de la TRR. En 2011, la SLANH, trabajando muy estrechamente con la Organización Panamericana de la Salud (OPS), estableció la meta para el 2019 (incluida en el Plan Estratégico $2014 \mathrm{OPS}^{10}$ ) de que todos los países de la zona tuvieran una prevalencia mínima de 700 ppmp, meta que afortunadamente ha ido lográndose en un número de países que no la tenían. Para lograr esta meta, en países con limitaciones económicas como los 
nuestros, es necesario estimular las TRR más costo-efectivas. Es notable que en América Latina, como promedio, el uso del trasplante renal es mucho menor que el deseable por ser la TRR más costo-efectiva y que la diálisis peritoneal esté francamente subutilizada ${ }^{6}$. Por tanto, la SLANH se ha propuesto estimular el mayor empleo de las TRR más costo-efectivas y, en conjunto con la Asociación Centroamericana y del Caribe de Nefrología e Hipertensión, ha llevado a cabo reuniones de líderes de la Nefrología y Sociedades Nacionales de la zona, con el fin de analizar las barreras, retos y oportunidades del empleo de la diálisis peritoneal (República Dominicana) y del trasplante renal (El Salvador). Ambas reuniones han terminado con la publicación de la Declaración de La Romana. Diálisis Peritoneal, una Alternativa a la Epidemia de la Enfermedad Renal ${ }^{11}$ y la Declaración de San Salvador. Trasplante Renal, la alternativa terapéutica más económica para la Enfermedad Renal Crónica ${ }^{12}$ en la que se plasman las conclusiones de dichas reuniones.

Una de las razones de que la diálisis peritoneal no sea usada con la frecuencia que sería aconsejable en Latinoamérica es la falta de conocimiento del personal nefrológico en esta forma de TRR. Los pacientes en diálisis peritoneal también son vistos por diferentes motivos en el primer nivel de atención, y el adecuado diagnóstico y manejo inicial de las complicaciones, así como la referencia oportuna al especialista, son muy importantes para lograr buenos resultados clínicos. Para lo anterior, los equipos multidisciplinarios necesitan conocer la diálisis peritoneal; además, si estos equipos de atención primaria recibieran entrenamiento en esta terapia, se podría informar sin sesgos a los pacientes que están próximos al inicio de diálisis e influir positivamente para que, cuando esté indicada, el paciente pueda seleccionar la diálisis peritoneal como TRR. Por tal motivo, la SLANH en colaboración estrecha con la OPS, ha desarrollado un Curso Virtual de Diálisis Peritoneal para el Equipo Multidisciplinario de Atención Primaria, que tiene las mismas características de desarrollo tecnológico y educativo que otro curso de prevención de la ERC que será detallado más adelante. Este curso, además de ser de enorme calidad, haber sido organizado por los coordinadores del Comité de Diálisis Peritoneal SLANH, e impartido por verdaderos expertos latinoamericanos, es gratuito y ha sido recientemente abierto para el público ${ }^{13}$.

Por otro lado, para poner en escena la enfermedad renal, y convencer a las autoridades de que es necesario lograr la cobertura universal de la TRR, instaurar legalmente y darle carácter obligatorio a los registros nacionales de diálisis y trasplantes, y avanzar en la consecución de planes nacionales de salud renal, se tienen que hacer labores de cabildeo y promoción ante autoridades gubernamentales. Con este fin, la SLANH, en apoyo de autoridades de algunas Sociedades Nacionales de Nefrología, ha tenido reuniones, por ejemplo, con el Senado de la República Mexicana y con la Presidencia de la República Dominicana. Este tipo de reuniones son necesarias para contribuir a lograr las metas.

\section{Reto: Alcanzar una tasa de 20 nefrólogos por millón de población}

Para alcanzar las metas propuestas y afrontar los retos arriba señalados, se requiere que existan un número adecuado de nefrólogos en cada país, para que sean los encargados de coordinar las acciones y los equipos de trabajo. Desafortunadamente, no es el caso en muchos países latinoamericanos, cuya tasa de nefrólogos es de las más bajas del mundo ${ }^{14}$. La SLANH no tiene una injerencia directa en la normatividad de los países para formar y regular la especialidad de la Nefrología, pero sí ha establecido las competencias y requerimientos mínimos que los especialistas en la materia debieran de cumplir ${ }^{15}$, y estimula, en cada una de sus actividades, la incorporación de gente nueva a la especialidad y a la sociedad. El número de especialistas ha ido creciendo a través de los años, y del 2014 al 2016 se notó un incremento en el promedio de nefrólogos de la región (14 y 16 pmp, respectivamente) ${ }^{6}$; sin embargo, las grandes disparidades aún son notables, y se observa un menor número de nefrólogos sobre todo en Centroamérica (justo donde existen las mayores necesidades e incidencia de problemas renales).

Para estimular la formación de nefrólogos en los países donde más se carece de ellos, así como mantener al día los conocimientos y la capacidad de los profesionales de la Nefrología, la SLANH mantiene, asociada con la International Society of Nephrology (ISN), los Programas de Fellowship y el de Educational Ambassador, específicos para llevar a cabo intercambios entre países de Latinoamérica, avalados por SLANH e ISN ${ }^{16}$. Estos programas aún pueden utilizarse más para contribuir a cumplir metas como las relacionadas con educación. 
Reto: Estimular la investigación local y regional sobre las causas de la enfermedad renal más comunes y particulares en América Latina

Los países de Latinoamérica sufren una notable transición socioeconómica y de problemas de salud ${ }^{1-5}$, con un notable incremento de las ECNT. En el caso de la $E R C$, las principales causas corresponden a las mismas ECNT observadas en otras partes del mundo: la diabetes mellitus y la hipertensión arterial ${ }^{9}$. En nuestra región, alrededor de $36 \%$ de los pacientes con TRR tienen a la diabetes mellitus como causa de insuficiencia renal crónica terminal $(\mathrm{IRCT})^{6}[\sim 70 \%$ en México y Puerto Rico ${ }^{9}$.

Además de las causas tradicionales, en Latinoamérica también se ha identificado una causa no tradicional de ERC, la llamada nefropatía mesoamericana o ERC de las comunidades agrícolas, cuya etiología aún no está completamente entendida (aunque se han propuesto varias hipótesis), que originalmente se describió que afectaba principalmente a hombres jóvenes que trabajaban en situaciones desventajosas en las labores agrícolas de determinadas áreas de la América Central. Aunque se han hecho importantes avances en su conocimiento ${ }^{17-19}$, aún hay una necesidad de apoyo a la investigación local y regional en esta entidad en particular, así como también hay necesidad de investigación en otras áreas importantes de la nefrología.

\section{Reto: Enfocarse en la prevención, diagnóstico y tratamiento de la enfermedad renal crónica temprana}

Para combatir la pandemia de la IRCT, es crucial prevenir y detectar en forma temprana a los pacientes con ERC y optimizar su cuidado para poder evitar el desarrollo de la enfermedad o enlentecer la progresión de la falla renal, prevenir sus consecuencias negativas, y disminuir la gran morbilidad y la mortalidad asociadas, además de disminuir los extraordinarios costos económicos de las TRR. Es indispensable, entonces, desarrollar programas de salud renal para la prevención, diagnóstico temprano y tratamiento oportuno 7 . También es extremadamente necesario implementar intervenciones educativas tanto para profesionales de la salud como para pacientes, particularmente en el primer nivel de atención ${ }^{20}$. Con la educación a los profesionales de atención primaria y a los pacientes, no solo se logra incrementar la competencia clínica de los profesionales en relación con la $E R C^{21}$, sino también se logra que los pacientes sean detectados y tratados más eficientemente y que incorporen conductas saludables de estilo de vida, y que se obtengan resultados favorables en su salud renal y general ${ }^{21,22}$. El empleo de modelos multidisciplinarios de salud renal se debe estimular en todo lugar en que sea posible su utilización, pues como se ha demostrado, estos modelos logran mejores resultados que el empleo de modelos convencionales de atención médica ${ }^{23,24}$.

Con el fin de incrementar la competencia clínica de los profesionales multidisciplinarios del primer nivel de atención, la SLANH desarrolló, en asociación con la OPS, un curso en línea masivo y gratuito (MOOC) sobre la Prevención y Manejo de la ERC para el Equipo Multidisciplinario de la Atención Primaria de la Salud, el cual se encuentra disponible en el campus virtual de salud pública de la OPS ${ }^{25}$. Este curso ha resultado muy exitoso, pues a octubre de 2018 tenía 16,000 alumnos inscritos de toda Latinoamérica y de otras partes del mundo, con una participación importante no solo de médicos de atención primaria, sino también de estudiantes, enfermeras, nutricionistas y de otras profesiones de la salud.

En ese mismo sentido, con el fin de incrementar el conocimiento sobre ERC del personal multidisciplinario, la ISN, a través de su ISN Academy ${ }^{26}$ y en colaboración con la SLANH, ha instituido una serie de dos a tres seminarios en red (webinarios) por año, que han empezado en mayo del 2018, y que continuarán por lo menos durante los 2 años siguientes. Estos webinarios son en español, su inscripción es gratis, y son impartidos y coordinados por expertos de la SLANH.

\section{Reto: Crear más registros de enfermedad renal en los países de Latinoamérica e incrementar la calidad de los ya existentes}

Una meta definida de la SLANH desde hace varios años ha sido que todo país de la región tenga un Registro Nacional de Diálisis y Trasplante, y que los que ya cuenten con él lo incrementen en calidad y confiabilidad. Para lograr este fin, la SLANH junto con la OPS, ha realizado desde hace varios años una serie de talleres en los que están presentes al menos un representante del Ministerio de Salud (invitado y apoyado por la OPS) y un representante de la Sociedad Nacional de Nefrología (invitado y apoyado por la SLANH). Estos talleres, realizados en diferentes países de Latinoamérica, tienen un enfoque regional, y se invita a los países vecinos de la sede en turno. De 2014 
a la fecha se han realizado 12 talleres, y en el penúltimo (llevado a cabo en la Ciudad de Panamá, Panamá), la mayor parte de países acudieron y expusieron el estado actual de su registro, identificando logros, dificultades y metas. En esta reunión pudo conocerse que a partir de que estos talleres se iniciaron se ha consolidado la creación oficial (con reconocimiento gubernamental) de registros nacionales en Ecuador, Costa Rica, El Salvador, Honduras, Panamá, República Dominicana y Paraguay, y se prevé que próximamente Perú también cuente con su registro nacional oficial. Aunque existe aún un gran camino por recorrer, pues aún hay países sin registro nacional o con un registro parcial, y la calidad de los mismos no es completamente confiable en todos, los avances logrados nos estimulan a seguir con esos esfuerzos.

\section{Colaboración con organismos internacionales}

Muchas de las acciones anteriores, la SLANH las ha logrado estableciendo alianzas con otras organizaciones internacionales con intereses afines. Por ejemplo, durante el World Congress of Nephrology 2017, llevado a cabo en la Ciudad de México, que fue organizado por la ISN y la SLANH como sociedad afiliada regional, se desarrolló el Primer Foro Global de Políticas en Salud Renal. En esta reunión estuvieron presentes el secretario de Salud de México y autoridades de ministerios de salud de otros países latinoamericanos invitados, autoridades de la ISN y del comité editorial de la revista Lancet, así como representantes de la SLANH, de organizaciones de pacientes renales y líderes de la nefrología regional. Las conclusiones de ese foro, que son extremadamente relevantes y que fueron acordadas $y$ firmadas por todos los participantes al evento, incluyen de alguna forma muchos de los puntos arriba expuestos, y se encuentran disponibles libremente en línea ${ }^{27}$.

Asimismo, la colaboración con la OPS ha sido discutida previamente, pero vale la pena recalcar que la SLANH es desde 2014 una organización no gubernamental en relaciones oficiales con la OPS, y como tal, además de colaborar en diversos puntos relativos a la enfermedad renal, ha sido invitada a conferencias y sesiones del Comité Ejecutivo y Regional de la OPS, y ha tenido la oportunidad de tomar la voz para llamar la atención de los representantes de los ministerios de salud de todas las Américas acerca de la gravedad del problema de la enfermedad renal en nuestra región. Afortunadamente, los frutos de todo este esfuerzo han ido apareciendo, pues varios gobiernos de países latinoamericanos empiezan a reconocer el problema y a tomar acciones para su solución, al igual que muchas sociedades en su conjunto.

En resumen, los principales retos en la atención de la ERC hoy día son:

- Lograr que haya cobertura universal de las TRR para que todos los pacientes que las requieren las reciban efectivamente.

- Lograr la meta de que al menos 700 ppmp sean tratados con TRR en todos los países de la región. Para ello, es necesario incentivar el mayor uso de las TRR costo-efectivas: trasplante renal y diálisis peritoneal.

- Estimular la mayor formación de nefrólogos en la zona para alcanzar una tasa de 20 por millón de población, pues para alcanzar las metas propuestas en el combate de la ERC, la participación central de este especialista es indispensable.

- Estimular la investigación local, regional y multicéntrica de calidad, sobre múltiples áreas de la ERC, incluyendo sus causas más comunes y las particulares que afectan a ciertas regiones. Los resultados de esta investigación contribuirán a resolver nuestros propios problemas.

- Contrarrestar efectivamente la pandemia de la ERC en América Latina no será posible si los esfuerzos no se enfocan en la prevención, diagnóstico y tratamiento de ERC temprana como objetivos principales. Incrementar la competencia clínica de los profesionales de la salud y la información y participación de los pacientes mediante intervenciones educativas es una de las mejores herramientas para este fin. Además, involucrar al personal multidisciplinario en modelos de intervención múltiple debe privilegiarse cuando sea posible.

- Para conocer la magnitud real de la ERC en nuestra región, poder tomar decisiones para contrarrestarla y evaluar los resultados de posibles acciones tomadas, es indispensable crear registros nacionales de ERC en todos los países que aún carezcan de ellos, e incrementar la calidad de los ya existentes.

\section{Conflicto de intereses}

El autor declara que no existe ningún conflicto de intereses.

\section{Responsabilidades éticas}

Protección de personas y animales. El autor declara que para esta investigación no se han realizado experimentos en seres humanos ni en animales. 
Confidencialidad de los datos. El autor declara que ha seguido los protocolos de su centro de trabajo sobre la publicación de datos de pacientes.

Derecho a la privacidad y consentimiento informado. El autor declara que en este artículo no aparecen datos de pacientes.

\section{Bibliografía}

1. Organización Panamericana de la Salud. Indicadores básicos. http:// www.paho.org/data/index.php/es/indicadores.html (Último acceso 23 septiembre de 2018).

2. Comisión Económica para América Latina y el Caribe (CEPAL). CEPALSTAT. http://estadisticas.cepal.org/cepalstat/web_cepalstat/estadisticaslndicadores.asp?idioma=e (Último acceso 20 de septiembre de 2018)

3. United Nations Educational, Scientific and Cultural Organization (UNESCO). http://www.unesco.org/new/en/unesco/worldwide/latin-america-and-the-caribbean/(Último acceso 21 de septiembre de 2018).

4. United Nations Development Programme. Human Development Reports. http://hdr.undp.org/sites/default/files/2018 summary human development statistical_update_sp.pdf (Último acceso 3 de noviembre de 2018).

5. World Bank. http://databank.bancomundial.org/data/reports.aspx?source=2\&country= LCN (Último acceso 3 de noviembre de 2018).

6. Sociedad Latinoamericana de Nefrología e Hipertensión. Registro Latinoamericano de Diálisis y Trasplante. http://slanh.net/wp-content/ uploads/2018/10/INFORME-2015-2016.pdf (Último acceso 10 de noviembre de 2018).

7. Cueto-Manzano AM, Douthat W. Lancet 2017 http://www.thelancet.com/ campaigns/kidney/updates/kidney-disease-in-latin-america (Último acceso 10 de noviembre de 2018)

8. Institute for Health Metrics and Evaluation. http://www.healthdata.org/ data-visualization/gbd-compare (Último acceso 9 de noviembre de 2018).

9. United States Renal Data System. https://www.usrds.org/2018/view/ v2 11.aspx (Último acceso 10 noviembre de 2018).

10. Plan Estratégico de la Organización Panamericana de la Salud 2014-2019. https://www.paho.org/hq/dmdocuments/2013/CE152-10-s.pd (Último acceso 9 de septiembre de 2018).

11. Sociedad Latinoamericana de Nefrología e Hipertensión. Declaración de la Romana "Diálisis Peritoneal, una Alternativa a la Epidemia de la Enfermedad Renal". http://slanh.net/declaracion-de-la-romana-dialisis-peritoneal-una-alternativa-a-la-epidemia-de-la-enfermedad-renal/(Último acceso 30 de agosto de 2018).

12. Sociedad Latinoamericana de Nefrología e Hipertensión. Declaración de San Salvador "Trasplante Renal, la Alternativa Terapéutica Más económica para la Enfermedad Renal Crónica". http://slanh.net/declaracion-centroamericana-y-del-caribe-de-trasplante-renal/(Último acceso 30 de agosto de 2018)
13. Curso Diálisis Peritoneal para el Equipo Multidisciplinario del Primer Nivel de Atención SLANH-OPS. Campus Virtual de Salud Pública de la OPS. https://mooc.campusvirtualsp.org/enrol/index.php?id=80 (Último acceso 11 de noviembre 2018).

14. Bello AK, Levin A, Tonelli M, Okpechi IG, Feehally J, Harris D, et al. Assessment of Global Kidney Health Care Status. JAMA. 2017;317:1864-81.

15. Di Bernardo JJ, editor. Recomendaciones para la formación de nefrólogos en Latinoamérica: Especialidad Nefrología de Adultos. Buenos Aires: Publicaciones Latinoamericanas SRL; 2015.

16. International Society of Nephrology. ISN Programs. https://www.theisn. org/programs/apply (Último acceso 11 de noviembre de 2018).

17. Kupferman J, Ramírez-Rubio O, Amador JJ, López-Pilarte D, Wilker EH, Laws RL, et al. Acute kidney injury in sugarcane workers at risk for Mesoamerican nephropathy. Am J Kidney Dis. 2018;72:475-82.

18. García-Trabanino R, Jarquín E, Wesseling C, Johnson RJ, González-Quiroz M, Weiss I, et al. Heat stress, dehydration, and kidney function in sugarcane cutters in El Salvador--A cross-shift study of workers at risk of Mesoamerican nephropathy. Environ Res. 2015;142:746-55.

19. Jayasumana C, Orantes C, De Broe ME. Chronic Interstitial Nephritis in Agricultural Communities. En: Turner NN, Lameire N, Goldsmith DJ, Winearls CJ, Himmelfarb J, Remuzzi G, et al., editors. Oxford Textbook of Clinical Nephrology. $4^{\text {th }}$ ed. Oxford University Press 2018.

20. Cueto-Manzano AM, Martínez-Ramírez HR, Cortés-Sanabria L, Rojas Campos E. CKD Screening and Prevention Strategies in Disadvantaged Populations. 31. The Role of Primary Health Care Professionals. En: García-García G, Agodoa LY, Norris KC, editors. Chronic Kidney Disease in Disadvantaged Populations. London: Elsevier Inc, 2017; p. 329-37. ISBN: 978-0-12-804311-0.

21. Cortés-Sanabria L, Cabrera-Pivaral CE, Cueto-Manzano AM, Rojas-Campos E, Barragán G, Hernández-Anaya M, et al. Improving care of patients with diabetes and CKD: A pilot study for a cluster-randomized trial. Am J Kidney Dis. 2008;51(5):777-88.

22. Cueto-Manzano AM, Martínez-Ramírez HR, Cortés-Sanabria L. Management of Chronic Kidney Disease: Primary Health-Care Setting, Self-Care and Multidisciplinary Approach. Clin Nephrol. 2010;74(Suppl 1): S99-S104.

23. Cueto-Manzano AM, Martínez-Ramírez HR, Cortés-Sanabria L. Comparison of primary health-care models in the management of chronic kidney disease. Kidney Int Supplements. 2013:3:210-4.

24. Martínez-Ramírez HR, Cortés-Sanabria L, Rojas-Campos E, Hernández-Herrera E, Cueto-Manzano AM. Multidisciplinary strategies in the management of early chronic kidney disease. Arch Med Res. 2013;44 (8): 611-5.

25. Curso Prevención y Manejo de la Enfermedad Renal Crónica para Equipos del Primer Nivel de Atención. Campus Virtual de Salud Pública de la OPS. https://mooc.campusvirtualsp.org/enrol/index.php?id=13 (Último acceso 10 de septiembre de 2018)

26. International Society of Nephrology. ISN Academy -Online Learning. https://academy.theisn. org/isn/\#! " menu $=16^{*}$ browseby $=9^{*}$ sortby $=1^{*}$ trend=12566 (Último acceso 11 de noviembre de 2018).

27. Sociedad Latinoamericana de Nefrología e Hipertensión. The Road to Global Kidney Health. Summary and Conclusions. http://slanh.net/theroad-to-global-kidney-health/(Último acceso 30 de julio de 2018). 